\title{
Proteinuria can predict prognosis after liver transplantation
}

\author{
Heng-Chih Pan ${ }^{1}$, Ying-Jen Chen², Jhe-Ping Lin ${ }^{3}$, Ming-Jung Tsai ${ }^{3}$, Chang-Chyi Jenq ${ }^{3,4}$, Wei-Chen Lee ${ }^{3,5^{*}}$, \\ Ming-Hung Tsai ${ }^{3,6}$, Pei-Chun Fan ${ }^{4}$, Chih-Hsiang Chang ${ }^{4}$, Ming-Yang Chang ${ }^{3,4}$, Ya-Chung Tian ${ }^{3,4}$, \\ Cheng-Chieh Hung ${ }^{3,4}$, Ji-Tseng Fang ${ }^{3,4}$, Chih-Wei Yang ${ }^{3,4}$ and Yung-Chang Chen ${ }^{3,4^{*}}$ (D)
}

\begin{abstract}
Background: Proteinuria is a manifestation of renal dysfunction and it has been demonstrated to be a significant prognostic factor in various clinical situations. The study was designed to analyze prognosis of patients receiving liver transplantation as well as to determine predictive performance of perioperative proteinuria.

Methods: We retrospectively reviewed data of patients who had received a liver transplant in a medical center between 2002 and 2010. Demographic information and clinical characteristic parameters were recorded on the day of intensive care unit admission before operation and on postoperative days 1, 7, and 14 .

Results: Among a total of 323 patients, in-hospital mortality and 90-day mortality rates were $13.0 \%(42 / 323)$ and $14.2 \%(46 / 323)$, respectively. Patients with proteinuria on admission had higher rates of acute kidney injury (26.8\% vs. $8.8 \%, p<0.001$ ), severe infection episodes ( $48.8 \%$ vs. $30.7 \%, p=0.023)$, hospital death $(31.1 \%$ vs. $10.1 \%$, $p<0.001$ ), and 90 -day mortality ( $37.7 \%$ vs. $10.9 \%, p<0.001)$. Multivariate analysis showed that proteinuria on admission and Sequential Organ Failure Assessment (SOFA) score were independent predictors of in-hospital mortality. The discriminatory ability of proteinuria plus SOFA was even better than that of SOFA alone, especially on postoperative day 1.

Conclusions: The presence of proteinuria before liver transplantation is supposed to be recognized as a negative predictor for in-hospital survival. Moreover, the presence of proteinuria after liver transplantation can assist in the early prediction of poor short-term prognosis for patients receiving liver transplantation.
\end{abstract}

Keywords: Liver transplantation, Proteinuria, SOFA, Prognosis, Mortality

\section{Background}

Proteinuria has been suggested to be a predictive factor and an important tool for differentiating the etiology of renal dysfunction in various clinical scenarios $[1,2]$. The good predictive performance of preoperative proteinuria utilized for the development of renal failure after operation has been reported [3]. Lin et al. also demonstrated that the presence of proteinuria in patients with end-stage liver disease is associated with increased risk of intensive care unit (ICU) mortality and poor shortterm outcome [4].

In the literature, prognostic significance of several scoring systems for end-stage liver disease has been

\footnotetext{
*Correspondence: weichen@cgmh.org.tw; cyc2356@gmail.com

${ }^{3}$ Chang Gung University College of Medicine, Taoyuan, Taiwan

Full list of author information is available at the end of the article
}

validated [5-7]. Wong and colleagues further compared the predictive accuracy of the commonly used scores in 149 end-stage liver disease patients undergone liver transplantation. The Sequential Organ Failure Assessment (SOFA) system was found to be superior to ChildPugh points (CP points) and Model for End-Stage Liver Disease (MELD) score, and postoperative day 7 SOFA had the best discriminative power for predicting 3-month and 1-year mortality after liver transplantation [8].

Renal dysfunction is one of the most significant adverse events in patients awaiting or undergoing a liver transplant, and its occurrence generally indicates a high rate of poor prognosis $[9,10]$. Prediction of acute kidney 
injury (AKI) is important for clinical decision making. Numerous researches have demonstrated that the presence of proteinuria provides a clue to the structural impairment of the kidney and reflects increased risk of developing AKI in general population [11, 12]. In spite of the fact that proteinuria has been increasingly considered as a significant manifestation of acute or chronic renal disease [13], no study clarify the association between presence of proteinuria and prognosis of patients undergoing liver transplant. This study aims to assess proteinuria as an early marker of renal dysfunction for liver transplant as well as to compare the outcome prediction efficacy of proteinuria with that of the main scores in the setting of liver transplant.

\section{Methods}

\section{Patient information and data collection}

This research was performed from October 2002 to December 2010 in a 2000-bed medical center in Taiwan. A total of 323 patients with end-stage liver disease and acute liver failure received liver transplant were included. Patients less than 18 years of age, patients who had previously received liver transplant, and patients with end-stage renal disease were excluded.

We retrospectively reviewed following data: demographic information, aetiologies of primary liver disease, clinical parameters, donor type, anesthesia time, operation time, duration of hospitalization and ICU stay, and outcome. Illness severity was evaluated on the day of ICU admission before operation and on postoperative day 1,7 , and 14 . The primary outcome of this research was in-hospital mortality rate. Follow-up at 90-day after transplantation was performed by chart records review or telephone interview [8].

\section{Definitions}

Urine dipstick analysis was used to detect proteinuria. The results were graded as negative (less than $15 \mathrm{mg} / \mathrm{dL}$ ), trace (15 to $30 \mathrm{mg} / \mathrm{dL}), 1+(30$ to $100 \mathrm{mg} / \mathrm{dL}), 2+$ (100 to $300 \mathrm{mg} / \mathrm{dL}$ ), $3+$ (300 to $1000 \mathrm{mg} / \mathrm{dL}$ ) or $4+$ (more than $1000 \mathrm{mg} / \mathrm{dL}$ ). In this study, proteinuria was defined as presence (urine dipstick reading trace, or $\geq 1+$ ) or absence (negative urine dipstick reading). The urinary analysis was performed on ICU admission and postoperative days 1, 7, and 14 [4].

Severity of liver disease was assessed by CP points and MELD score $[14,15]$. Severity of illness was also graded by SOFA score according to the six organ systems. AKI was diagnosed according to definition of the RIFLE criteria (Table 1). The RIFLE classification was also used to classify patients into risk (RIFLE-R), injury (RIFLE-I), and failure (RIFLE-F) groups [16].
No patient met the criteria for classification of loss of kidney function (RIFLE-L) or end-stage renal disease (RIFLE-E). The following simple model for mortality was constructed: non-acute kidney injury (0 points), RIFLE-R (1 point), RIFLE-I ( 2 points), and RIFLE-F (3 points) [15]. The worst values measured on the day of ICU admission and postoperative days 1, 7, and 14 were recorded.

\section{Statistical analysis}

Data analyses were performed using the statistical package SPSS 19.0 (SPSS, Inc., Chicago, IL, USA). All statistical tests are 2-tailed. A $p$-value of $<0.05$ is considered to represent statistical significance. Continuous variables were presented as means and standard derivations, and categorical data were summarized as frequency and percentage unless otherwise stated. Hospital survivors were compared with nonsurvivors in the primary analysis. Kolmogorov-Smirnov test was employed for testing normal distribution. Normally distributed continuous variables were compared by Student's $t$-test and non-normally distributed ones were compared by Mann-Whitney $U$ test. Categorical data were tested by the chi-square test. The risk factors for in-hospital mortality were assessed by univariate analysis, and statistically significant variables were included in the multivariate analysis. For analyzing these variables, backward multiple logistic regression model was employed.

Hosmer-Lemeshow goodness-of-fit test was used to examined calibration and compare the number of predicted and observed mortality. Discrimination in predicting 90-day mortality was assessed by area under the receiver operating characteristic (AUROC) curve. Nonparametric approach was used to compare the AUROC values. Analyses of the ROC curves were applied for calculating sensitivity, specificity, and overall correctness. The cutoff value was decided according to the ability to offer the highest Youden index [17]. Cumulative survival curves were plotted using the Kaplan-Meier method and compared by the $\log$ rank test. Correlation of proteinuria and serum creatinine (SCr) measured on ICU admission was measured by Pearson analysis and linear regression. The prevalence of proteinuria before operation, and on postoperative day 1,7 , and 14 were compared between 90 -day survivors and nonsurvivors by repeated-measures analysis of variance using the general linear model procedure.

\section{Results}

\section{Patient characteristics}

Three hundred and twenty-three patients who received liver transplant from October 2002 to December 2010 were enrolled. Overall in-hospital mortality rate was $13.0 \%(42 / 323)$. Table 2 listed patient data and 
Table 1 The criteria of SOFA score and RIFLE classification

\begin{tabular}{|c|c|c|c|c|c|}
\hline SOFA Score & 0 & 1 & 2 & 3 & 4 \\
\hline \multicolumn{6}{|l|}{ Respiration } \\
\hline $\mathrm{PaO} 2 / \mathrm{FiO}_{2}$ & $>400$ & $>300-\leq 400$ & $>200-\leq 300$ & $>100-\leq 200$ with ventilator & $\leq 100$ with ventilator \\
\hline \multicolumn{6}{|l|}{ Coagulation } \\
\hline Platelets, $\times 10^{3} / \mathrm{mm}^{3}$ & $>150$ & $>100-\leq 150$ & $>50-\leq 100$ & $>20-\leq 50$ & $\leq 20$ \\
\hline \multicolumn{6}{|l|}{ Liver } \\
\hline Bilirubin, $\mathrm{mg} / \mathrm{dL}(\mu \mathrm{mol} / \mathrm{L})$ & $<1.2(<20)$ & $\geq 1.2-<2.0(20-32)$ & $\geq 2.0-<6.0(33-101)$ & $\geq 6.0-<12.0(102-204)$ & $\geq 12.0(>204)$ \\
\hline \multicolumn{6}{|l|}{ Cardiovascular } \\
\hline Hypotension & $\mathrm{MAP} \geq 70 \mathrm{~mm} \mathrm{Hg}$ & MAP $<70 \mathrm{~mm} \mathrm{Hg}$ & $\begin{array}{l}\text { Dopamine } \leq 5 \text { or } \\
\text { dobutamine (any dose) }\end{array}$ & $\begin{array}{l}\text { Dopamine }>5 \text { or epi } \leq 0.1 \\
\text { or norepi } \leq 0.1^{\text {a }}\end{array}$ & $\begin{array}{l}\text { Dopamine }>15 \text { or epi }>0.1 \\
\text { or norepi }>0.1^{\text {a }}\end{array}$ \\
\hline \multicolumn{6}{|l|}{ CNS } \\
\hline Glasgow Coma Score & 15 & $13-14$ & $10-12$ & $6-9$ & $<6$ \\
\hline \multicolumn{6}{|l|}{ Renal } \\
\hline $\begin{array}{l}\text { Creatinine, } \mathrm{mg} / \mathrm{dL}(\mu \mathrm{mol} / \mathrm{L}) \\
\text { or urine output }\end{array}$ & $<1.2(<110)$ & $\geq 1.2-<2.0(110-170)$ & $\geq 2.0-<3.5(171-299)$ & $\begin{array}{l}\geq 3.5-<5.0(300-440) \\
\text { or }<500 \mathrm{~mL} / \text { day }\end{array}$ & $\geq 5.0(>440)$ or $<200 \mathrm{~mL} /$ day \\
\hline RIFLE Classification & \multicolumn{4}{|l|}{ SCr criteria } & UO Criteria \\
\hline Definition & \multicolumn{4}{|c|}{ SCr changes over 1-7 days, sustained for more than $24 \mathrm{~h}$} & $\mathrm{UO}<0.5 \mathrm{ml} / \mathrm{kg} / \mathrm{h} \times 6 \mathrm{~h}$ \\
\hline Risk & \multicolumn{4}{|c|}{ Increase in $\mathrm{SCr} \geq 1.5 \times$ baseline or decrease in GFR $\geq 25 \%$} & $\mathrm{UO}<0.5 \mathrm{ml} / \mathrm{kg} / \mathrm{h} \times 6 \mathrm{~h}$ \\
\hline Injury & \multicolumn{4}{|c|}{ Increase in $\mathrm{SCr} \geq 2.0 \times$ baseline or decrease in $\mathrm{GFR} \geq 50 \%$} & $\mathrm{UO}<0.5 \mathrm{ml} / \mathrm{kg} / \mathrm{h} \times 12 \mathrm{~h}$ \\
\hline Failure & \multicolumn{4}{|c|}{$\begin{array}{l}\text { Increase in } \mathrm{SCr} \geq 3.0 \times \text { baseline or an absolute serum creatinine } \geq 4.0 \mathrm{mg} / \mathrm{dl} \\
\text { with an acute rise of at least } 0.5 \mathrm{mg} / \mathrm{dl} \text { or decrease in GFR } \geq 75 \%\end{array}$} & $\begin{array}{l}\mathrm{UO}<0.5 \mathrm{ml} / \mathrm{kg} / \mathrm{h} \times 24 \mathrm{~h} \\
\text { or anuria } \times 12 \mathrm{~h}\end{array}$ \\
\hline Loss & \multicolumn{5}{|c|}{ Complete loss of kidney function $>4$ weeks } \\
\hline ESRD & \multicolumn{5}{|c|}{ End-stage renal disease ( $>3$ months) } \\
\hline
\end{tabular}

abbreviations: SOFA the sequential organ failure assessment, RIFLE risk of renal failure, injury to kidney, failure of kidney function, loss of kidney function, and end-stage renal failure, SCr serum creatinine, UO urine output, hrs hours, ESRD end-stage renal disease, RRT renal replacement therapy 
clinical characteristics of both survivors and nonsurvivors. Mean patient age was 51 years; 231 were male (71\%) and 92 were female (29\%). Ninety-one patients (28.2 \%) received deceased-donor grafts. Mean length of ICU stay was 21 days. There was no significant difference in age or gender between survivors and nonsurvivors. Table 3 listed primary liver diseases and presumptive causes of AKI on the first day after transplantation. In this research, hepatitis B virus infection (34\%) was the leading cause of liver diseases, followed by hepatitis B virus infection with hepatoma (15\%). Patient who developed AKI tended to attribute to multiple reasons (23\%), followed by infection (13\%).

\section{Risk factors for adverse outcomes}

Table 4 listed the correlation of operation time and newly onset proteinuria after transplantation. Among patients who received deceased-donor grafts,

Table 2 Patient demographic data and clinical characteristics according to in-hospital mortality

\begin{tabular}{|c|c|c|c|c|}
\hline & All patients $(n=323)$ & Survivors $(n=281)$ & Non-survivors $(n=42)$ & $P$-value \\
\hline Age (years) & $50.8 \pm 10.4$ & $50.9 \pm 9.8$ & $50.3 \pm 13.8$ & NS (0.753) \\
\hline Gender (M/F) & $231 / 92$ & $199 / 82$ & $32 / 10$ & NS $(0.583)$ \\
\hline BMI on admission $\left(\mathrm{kg} / \mathrm{m}^{2}\right)$ & $24.3 \pm 4.0$ & $24.7 \pm 4.0$ & $21.1 \pm 2.4$ & $<0.001$ \\
\hline History of diabetes mellitus (yes/no) & $55 / 268$ & $46 / 235$ & $9 / 33$ & NS (0.387) \\
\hline History of chronic kidney disease (yes/no) & $31 / 292$ & $22 / 259$ & $9 / 33$ & 0.005 \\
\hline Proteinuria on admission (yes/no) & $45 / 278$ & $31 / 250$ & $14 / 28$ & $<0.001$ \\
\hline Hemoglobin on admission (g/dL) & $10.6 \pm 2.2$ & $10.7 \pm 2.2$ & $9.8 \pm 2.1$ & 0.008 \\
\hline Leukocytes on admission $\left(\times 10^{9} / \mathrm{L}\right)$ & $2.9 \pm 3.7$ & $2.8 \pm 3.5$ & $3.3 \pm 4.9$ & NS (0.569) \\
\hline Platelets on admission $\left(\times 10^{9} / \mathrm{L}\right)$ [median] & $72.7[60]$ & $72.9 \pm[60]$ & $71.1[59]$ & NS (0.809) \\
\hline Prothrombin time INR on admission & $1.8 \pm 0.7$ & $1.8 \pm 0.7$ & $1.9 \pm 0.7$ & NS (0.050) \\
\hline Serum sodium on admission (mmol/L) & $137.8 \pm 5.7$ & $137.9 \pm 5.4$ & $137.0 \pm 8.0$ & NS $(0.471)$ \\
\hline AST on admission (U/L) [median] & $88.8[62]$ & $87.5[64]$ & $98.3[51]$ & NS (0.498) \\
\hline ALT on admission (U/L) [median] & $67.2[39]$ & $67.4[40]$ & $65.8[34]$ & NS (0.938) \\
\hline Total bilirubin on admission ( $\mu \mathrm{mol} / \mathrm{L})$ [median] & $145.4[51]$ & $130.0[50]$ & $244.5[96]$ & 0.003 \\
\hline A-a gradient on admission $(\mathrm{mmHg})$ [median] & $25.1[17]$ & $22.8[17]$ & $43.3[18]$ & 0.039 \\
\hline Serum creatinine on admission ( $\mu \mathrm{mol} / \mathrm{L}$ ) [median] & $97.2[77]$ & $92.2[75]$ & $114.9[84]$ & NS $(0.064)$ \\
\hline Hepatorenal syndrome, n (\%) & $29(9)$ & $22(8)$ & $7(17)$ & NS (0.079) \\
\hline MAP on admission (mmHg) & $86.1 \pm 12.4$ & $86.3 \pm 12.7$ & $84.7 \pm 10.3$ & NS $(0.427)$ \\
\hline Child-Pugh points on admission & $10.1 \pm 2.6$ & $9.9 \pm 2.7$ & $11.1 \pm 2.0$ & 0.010 \\
\hline MELD score on admission & $17.6 \pm 9.0$ & $17.1 \pm 8.9$ & $21.4 \pm 9.7$ & 0.025 \\
\hline RIFLE on admission (No AKI/Risk/Injury/Failure) & $286 / 16 / 9 / 12$ & 250/13/9/9 & $36 / 3 / 0 / 3$ & NS (0.449) \\
\hline SOFA score on admission & $5.1 \pm 2.7$ & $4.8 \pm 2.5$ & $6.7 \pm 3.3$ & 0.001 \\
\hline Anesthesia time during operation (hours) & $12.1 \pm 1.8$ & $12.1 \pm 1.9$ & $12.4 \pm 1.5$ & NS $(0.362)$ \\
\hline Donor type (DDLT /LDLT) & $91 / 232$ & $74 / 207$ & $17 / 25$ & 0.018 \\
\hline Total operative time (mins) [median] & $687.1[683]$ & $685.6[683]$ & $697.2[682]$ & NS $(0.589)$ \\
\hline Cold ischemia time (mins) [median] & $85.0[15]$ & $74.8[15]$ & $166.4[17]$ & NS $(0.125)$ \\
\hline Warm ischemia time (mins) [median] & $128.2[122]$ & $126.3[121]$ & $141.1[129]$ & NS (0.115) \\
\hline Graft-to-recipient weight ratio (\%) & $1.0 \pm 0.3$ & $1.0 \pm 0.3$ & $1.1 \pm 0.5$ & NS (0.125) \\
\hline Blood loss volume during operation (ml) [median] & 3034 [2000] & 2672 [1840] & $4430[2000]$ & 0.014 \\
\hline Length of ICU stay (days) [median] & $21.0[14]$ & $19.2[14]$ & $33.6[24]$ & 0.002 \\
\hline Length of hospital stay (days) [median] & $47.8[38]$ & $46.7[38]$ & $54.7[44]$ & NS $(0.215)$ \\
\hline
\end{tabular}

Values in bold are statistically significant $(P$-value $<0.05)$

There were significant differences in BMI on admission, history of chronic kidney disease, presence of proteinuria on admission, hemoglobin on admission, total bilirubin on admission, A-a gradient on admission, Child-Pugh points on admission, MELD score on admission, SOFA score on admission, blood loss volume during operation, and length of ICU stay

Abbreviation: $M$ male, $F$ female, ICU intensive care unit, MAP mean arterial pressure, INR international normalized ratio, $A S T$ aspartate aminotransferase, $A L T$ alanine aminotransferase, DM diabetes mellitus, MELD model for end-stage liver disease, SOFA sequential organ failure assessment, $A K I$ acute kidney injury, DDLT deceased donor liver transplantation, LDLT living donor liver transplantation 
Table 3 Primary liver diseases and presumptive causes of AKI after operation according to in-hospital mortality

\begin{tabular}{|c|c|c|c|c|}
\hline & $\begin{array}{l}\text { All patients } \\
n=323\end{array}$ & $\begin{array}{l}\text { Survivors } \\
n=281\end{array}$ & $\begin{array}{l}\text { Non-survivors } \\
n=42\end{array}$ & $P$-value \\
\hline Primary liver disease & $323(100)$ & $281(100)$ & $42(100)$ & \\
\hline Alcoholic, n (\%) & $16(5)$ & $12(4)$ & $4(10)$ & NS (0.254) \\
\hline Hepatitis B, n (\%) & $111(34)$ & $100(36)$ & $11(26)$ & NS (0.848) \\
\hline Hepatitis C, n (\%) & $31(10)$ & $23(8)$ & $8(19)$ & 0.003 \\
\hline Hepatoma, n (\%) & $3(1)$ & $3(1)$ & $0(0)$ & NS (1.000) \\
\hline Alcoholic + hepatitis B, n (\%) & $21(6)$ & $15(7)$ & $2(5)$ & NS (1.000) \\
\hline Alcoholic + hepatitis C, n (\%) & $5(2)$ & $5(2)$ & $0(0)$ & NS (1.000) \\
\hline Alcoholic + hepatoma, n (\%) & $3(1)$ & $3(1)$ & $0(0)$ & NS (1.000) \\
\hline Hepatitis B + hepatitis C, n (\%) & $17(5)$ & $14(5)$ & $3(7)$ & NS (0.723) \\
\hline Hepatitis B + hepatoma, n (\%) & $49(15)$ & $43(15)$ & $6(14)$ & NS (0.172) \\
\hline Hepatitis C + hepatoma, n (\%) & $31(10)$ & $29(10)$ & $2(5)$ & NS (0.134) \\
\hline Alcoholic + hepatitis B + hepatoma, n (\%) & $2(1)$ & $1(1)$ & $1(2)$ & NS (0.429) \\
\hline Other causes, $\mathrm{n}(\%)^{\mathrm{a}}$ & $34(10)$ & $29(10)$ & $5(12)$ & NS (0.787) \\
\hline Presence of AKI after transplantation (Post-OP day 1 ) & $125(39)$ & $101(36)$ & $24(57)$ & 0.011 \\
\hline Prerenal type of AKI, n (\%) & $2(1)$ & $2(1)$ & $0(0)$ & NS (1.000) \\
\hline Infection related AKI, n (\%) & $42(13)$ & $31(11)$ & $11(26)$ & 0.006 \\
\hline Nephrotoxic agent exposure related AKI, n (\%) & $6(2)$ & $6(2)$ & $0(0)$ & NS (0.601) \\
\hline Mixed type and other causes of AKI, n (\%) ${ }^{\mathrm{b}}$ & $75(23)$ & $62(22)$ & $13(31)$ & NS (0.236) \\
\hline
\end{tabular}

Values in bold are statistically significant $(P$-value $<0.05)$

Hepatitis $C$ virus infection was independently associated with in-hospital mortality

Presence of infection related AKI on the first day after transplantation was independently associated with in-hospital mortality

${ }^{a}$ Biliary cirrhosis, biliary sclerosis, autoimmune hepatitis, Wilson's disease, polycystic liver disease, drugs, and unknown causes

${ }^{\mathrm{b}}$ Multifactor related, ischemia/reperfusion injury, or unknown cause

those with newly onset proteinuria tended to have longer cold ischemia time. While in patients who received living-donor grafts, those with newly onset proteinuria tended to have longer warm ischemia time.

The univariate analysis showed that 9 (Table 5) out of the 31 variables (Table 2) were good prognostic indicators for in-hospital mortality. On performing the multivariate analysis, we recognized presence of proteinuria and SOFA determined on the first day of ICU admission as having independent prognostic significance (Table 5). Regression coefficients of these variables were used to calculate the odds of death in each patient as follows:

Logarithm of odds of death $=-2.471+1.320 \times$ Proteinuria $+0.157 \times$ SOFA score.

Table 4 Operation time according to newly onset proteinuria after transplantation

\begin{tabular}{|c|c|c|c|c|}
\hline & $\begin{array}{l}\text { Patients with no proteinuria } \\
\text { on admission }\end{array}$ & $\begin{array}{l}\text { Patients with newly onset } \\
\text { proteinuria on post-OP day } 1\end{array}$ & $\begin{array}{l}\text { Patients with no newly onset } \\
\text { proteinuria on post-OP day } 1\end{array}$ & $P$-value \\
\hline LDLT & $n=208$ & $n=65(31.2 \%)$ & $n=143(68.8 \%)$ & \\
\hline Total operative time (mins) [median] & $703.9[695]$ & $789.3[811]$ & $707.6[691]$ & NS $(0.087)$ \\
\hline Cold ischemia time (mins) [median] & $85.4[14]$ & $27.9[15]$ & 21.9 [13] & NS (0.659) \\
\hline Warm ischemia time (mins) [median] & $128.2[130]$ & 194.0 [182] & 127.6 [133] & 0.004 \\
\hline DDLT & $n=70$ & $n=47(67.1 \%)$ & $n=23(32.9 \%)$ & \\
\hline Total operative time (mins) [median] & $640.0[633]$ & $646.2[653]$ & $625.8[632]$ & NS (0.876) \\
\hline Cold ischemia time (mins) [median] & $651.3[583]$ & $756.5[634]$ & $552.0[541]$ & 0.039 \\
\hline Warm ischemia time (mins) [median] & $113.5[91]$ & $114.0[90]$ & $101.4[90]$ & NS (0.737) \\
\hline
\end{tabular}

Values in bold are statistically significant $(P$-value $<0.05)$

In the LDLT group, patients with newly onset proteinuria had significantly longer warm ischemia time In the DDLT group, patients with newly onset proteinuria had significantly longer cold ischemia time Abbreviation: $D D L T$ deceased donor liver transplantation, $L D L T$ living donor liver transplantation 
Table 5 Variables showing prognostic significance for in-hospital mortality

\begin{tabular}{|c|c|c|c|c|}
\hline Parameters & Beta Coefficient & Standard error & Odds ratios $(95 \% \mathrm{Cl})$ & $P$-value \\
\hline \multicolumn{5}{|l|}{ Univariate logistic regression } \\
\hline BMI on admission $\left(\mathrm{kg} / \mathrm{m}^{2}\right)$ & -0.247 & 0.050 & $0.781(0.708-0.861)$ & $<0.001$ \\
\hline History of chronic kidney disease & 1.167 & 0.437 & $3.211(1.364-7.557)$ & 0.008 \\
\hline Proteinuria on admission & 1.480 & 0.398 & $4.391(2.011-9.587)$ & $<0.001$ \\
\hline Hemoglobin on admission (g/dL) & -0.218 & 0.083 & $0.804(0.683-0.947)$ & 0.009 \\
\hline Total bilirubin on admission (mg/dL) & 0.037 & 0.011 & $1.038(1.015-1.061)$ & 0.001 \\
\hline Donor type (DDLT /LDLT) & -0.41 & 0.169 & $0.664(0.477-0.925)$ & 0.015 \\
\hline A-a gradient on admission & 0.009 & 0.003 & $1.009(1.002-1.016)$ & 0.007 \\
\hline Child-Pugh points on admission & 0.176 & 0.070 & $1.193(1.040-1.368)$ & 0.012 \\
\hline MELD score on admission & 0.046 & 0.056 & $1.047(0.999-1.098)$ & NS (0.056) \\
\hline SOFA score on admission & 0.219 & 0.057 & $1.245(1.114-1.391)$ & $<0.001$ \\
\hline Blood loss volume during operation (ml) & $<0.001$ & $<0.001$ & $1.000(1.000-1.000)$ & 0.003 \\
\hline Length of ICU stay (days) & 0.018 & 0.006 & $1.018(1.007-1.029)$ & 0.002 \\
\hline \multicolumn{5}{|l|}{ Multivariate logistic regression } \\
\hline Proteinuria on admission & 1.320 & 0.478 & $3.745(1.468-9.554)$ & 0.006 \\
\hline SOFA on admission & 0.157 & 0.067 & $1.170(1.027-1.333)$ & 0.019 \\
\hline Constant & -2.471 & 0.245 & 0.085 & $<0.001$ \\
\hline
\end{tabular}

On performing multivariate logistic regression, the presence of proteinuria on admission and SOFA score on admission had independent prognostic significance for assessing in-hospital mortality

Abbreviation: MELD model for end-stage liver disease, SOFA sequential organ failure assessment

\section{Calibration and discrimination of the scoring systems}

Table 6 showed values of calibration and discrimination of proteinuria, CP points, MELD, RIFLE, and SOFA in predicting 90-day mortality. For assessing calibration, the number of observed and predicted mortality was compared by Hosmer-Lemeshow goodness-of-fit. Discriminatory power was assessed by AUROC. On basis of the ROC analysis, discriminatory ability of SOFA and MELD determined on preoperative, postoperative days 1,7 , and 14 were better than that of CP points and proteinuria.

The proteinuria plus SOFA score [following variables were applied for the calculation: presence of proteinuria (1 point) and SOFA] was defined as the addition of the two variables, with sum ranging from 0 to 25 [4]. The discriminatory ability of this score seemed to be superior to that of other evaluating systems, including proteinuria, CP points, MELD, RIFLE, SOFA, and proteinuria plus nonrenal SOFA scores. AUROC curves were highest for proteinuria plus SOFA on postoperative day 7 to predict 90-day mortality $(0.907 \pm 0.041)$. AUROC value for proteinuria plus SOFA determined on postoperative day 1 was significantly higher than that for proteinuria, RIFLE, CP points, and MELD. AUROC for proteinuria plus SOFA determined on postoperative day 7 and 14 were significantly higher than that for proteinuria, RIFLE, and CP points.

\section{Indices for predicting short-term prognosis}

For evaluation and validation of the scoring systems, we compared the sensitivity, specificity, and overall correctness of prediction at cut-off values which could offer the highest Youden index (Table 7). At preoperative, postoperative days 1,7 , and 14, The proteinuria plus SOFA had the best Youden index and overall correctness in predicting 90-day mortality

In this study population, 45 patients had proteinuria while 278 patients had no proteinuria on ICU admission. Patients with proteinuria on admission had higher incidence of AKI (26.8 \% vs. $8.8 \%, p<0.001)$, severe infection episodes requiring prolonged courses of antibiotics or inotropic agents ( $48.8 \%$ vs. $30.7 \%, p=0.023)$, hospital death (31.1\% vs. $10.1 \%, p<0.001)$, and 90 -day mortality $(37.7 \%$ vs. $10.9 \%, p<0.001)$ than those without proteinuria.

Figure 1 illuminates the significantly different cumulative survival rates between patients with and without proteinuria as well as the similar cumulative survival rates between patients with and without $\mathrm{SCr}$ level elevation (Increase in $\mathrm{SCr} \geq 1.5 \times$ baseline) on ICU admission (before transplantation). Fig. 2 shows the weak correlation of proteinuria and $\mathrm{SCr}$ measured on ICU admission $(P=0.143)$. Fig. 3 illustrates significant increases in the prevalence of proteinuria during the time (starting before transplantation to 14 days postoperatively) among hospital and 90-day mortality groups but not survival groups. 
Table 6 Calibration and discrimination for the scoring methods used in predicting 90-day mortality

\begin{tabular}{|c|c|c|c|c|c|c|}
\hline & \multicolumn{3}{|l|}{ Calibration } & \multicolumn{3}{|l|}{ Discrimination } \\
\hline & Goodness-of-fit $\left(x^{2}\right)$ & df & $p$ & $\overline{A U R O C} \pm \mathrm{SE}$ & $95 \% \mathrm{Cl}$ & $P$ \\
\hline \multicolumn{7}{|l|}{ On admission } \\
\hline Proteinuria & - & - & - & $0.582 \pm 0.053$ & $0.479-0.685$ & NS (0.100) \\
\hline Child-Pugh points & 10.157 & 7 & 0.180 & $0.580 \pm 0.041$ & $0.499-0.662$ & NS (0.087) \\
\hline MELD score & 5.845 & 8 & 0.665 & $0.609 \pm 0.067$ & $0.418-0.680$ & NS (0.067) \\
\hline RIFLE & - & - & - & $0.577 \pm 0.067$ & $0.355-0.618$ & NS (0.845) \\
\hline SOFA & 1.618 & 5 & 0.899 & $0.648 \pm 0.049$ & $0.552-0.745$ & 0.002 \\
\hline Proteinuria plus SOFA & 1.047 & 5 & 0.959 & $0.659 \pm 0.052$ & $0.557-0.761$ & 0.002 \\
\hline Proteinuria plus nonrenal SOFA & 6.347 & 6 & 0.385 & $0.658 \pm 0.055$ & $0.550-0.765$ & 0.002 \\
\hline \multicolumn{7}{|l|}{ Postoperative day 1} \\
\hline Proteinuria & - & - & - & $0.609 \pm 0.090$ & $0.432-0.786$ & NS (0.240) \\
\hline Child-Pugh points & 2.438 & 5 & 0.786 & $0.639 \pm 0.062$ & $0.479-0.721$ & NS (0.142) \\
\hline MELD score & 5.947 & 8 & 0.653 & $0.705 \pm 0.044$ & $0.620-0.791$ & $<0.001$ \\
\hline RIFLE & 2.684 & 2 & 0.261 & $0.626 \pm 0.048$ & $0.531-0.720$ & 0.007 \\
\hline SOFA & 3.063 & 8 & 0.930 & $0.761 \pm 0.043$ & $0.676-0.845$ & $<0.001$ \\
\hline Proteinuria plus SOFA & 7.406 & 6 & 0.285 & $0.828 \pm 0.062$ & $0.707-0.949$ & $<0.001$ \\
\hline Proteinuria plus nonrenal SOFA & 11.595 & 5 & 0.041 & $0.823 \pm 0.078$ & $0.670-0.977$ & 0.001 \\
\hline \multicolumn{7}{|l|}{ Postoperative day 7} \\
\hline Proteinuria & - & - & - & $0.757 \pm 0.056$ & $0.647-0.866$ & $<0.001$ \\
\hline Child-Pugh points & 6.365 & 4 & 0.173 & $0.750 \pm 0.065$ & $0.593-0.847$ & 0.001 \\
\hline MELD score & 26.161 & 8 & 0.001 & $0.856 \pm 0.038$ & $0.782-0.930$ & $<0.001$ \\
\hline RIFLE & 9.602 & 2 & 0.008 & $0.825 \pm 0.042$ & $0.742-0.908$ & $<0.001$ \\
\hline SOFA & 6.073 & 6 & 0.415 & $0.899 \pm 0.031$ & $0.838-0.961$ & $<0.001$ \\
\hline Proteinuria plus SOFA & 8.625 & 6 & 0.196 & $0.907 \pm 0.041$ & $0.825-0.988$ & $<0.001$ \\
\hline Proteinuria plus nonrenal SOFA & 7.856 & 6 & 0.249 & $0.903 \pm 0.038$ & $0.828-0.978$ & $<0.001$ \\
\hline \multicolumn{7}{|l|}{ Postoperative day 14} \\
\hline Proteinuria & - & - & - & $0.773 \pm 0.067$ & $0.642-0.904$ & 0.005 \\
\hline Child-Pugh points & 3.469 & 3 & 0.325 & $0.783 \pm 0.052$ & $0.682-0.885$ & $<0.001$ \\
\hline MELD score & 134.84 & 8 & $<0.001$ & $0.850 \pm 0.056$ & $0.740-0.960$ & $<0.001$ \\
\hline RIFLE & 1.658 & 2 & 0.436 & $0.780 \pm 0.050$ & $0.681-0.879$ & $<0.001$ \\
\hline SOFA & 24.495 & 7 & 0.001 & $0.892 \pm 0.044$ & $0.806-0.978$ & $<0.001$ \\
\hline Proteinuria plus SOFA & 3.987 & 7 & 0.781 & $0.900 \pm 0.042$ & $0.819-0.982$ & $<0.001$ \\
\hline Proteinuria plus nonrenal SOFA & 5.009 & 7 & 0.659 & $0.894 \pm 0.038$ & $0.819-0.969$ & $<0.001$ \\
\hline
\end{tabular}

Values in bold are statistically significant $(P$-value $<0.05)$

On ICU admission day (before transplantation): The prediction accuracy of the SOFA score was better than those of the Child-Pugh points, MELD score and RIFLE. The proteinuria plus SOFA score has an even better discriminatory power than the SOFA score

On post-transplant day 1, 7, 14: The prediction accuracy of the SOFA and MELD score was better than that of the Child-Pugh points. The proteinuria plus SOFA score has an even better discriminatory power than the SOFA score

Abbreviation: MELD, model for end-stage liver disease; SOFA, sequential organ failure assessment; df, degree of freedom; AUROC, areas under the receiver operating characteristic curve; SE, standard error; $\mathrm{Cl}$, confidence intervals; NS, not significant

\section{Discussion}

In the current research, overall hospital survival rate was $87.0 \%(281 / 323)$, which is in agreement with what had been presented in the literature $[8,18,19]$. Our investigation found that both presence of proteinuria and SOFA score determined on the first day of admission to the ICU were significantly correlated to in-hospital mortality (Tables 2 and 5). Analytical results also proved that discriminatory ability of SOFA was better than those of CP points, RIFLE, and MELD. One notable finding of this study was that presence of proteinuria in combination with SOFA has an even more superior discriminatory 
Table 7 Prediction of subsequent 90-day mortality

\begin{tabular}{|c|c|c|c|c|c|}
\hline Predictive factors & Cutoff point & Youden index & Sensitivity (\%) & Specificity (\%) & Overall correctness (\%) \\
\hline \multicolumn{6}{|l|}{ Proteinuria } \\
\hline On admission & positive & 0.16 & 28 & 88 & 58 \\
\hline Postoperative day 1 & positive & 0.22 & 64 & 58 & 61 \\
\hline Postoperative day 7 & positive & 0.51 & 85 & 66 & 76 \\
\hline Postoperative day 14 & positive & 0.55 & 90 & 65 & 77 \\
\hline \multicolumn{6}{|l|}{ Child-Pugh points } \\
\hline On admission & 12 & 0.17 & 95 & 22 & 59 \\
\hline Postoperative day 1 & 10 & 0.26 & 90 & 37 & 64 \\
\hline Postoperative day 7 & 9 & 0.42 & 60 & 82 & 71 \\
\hline Postoperative day 14 & 8 & 0.42 & 50 & 92 & 71 \\
\hline \multicolumn{6}{|l|}{ MELD score } \\
\hline On admission & 15 & 0.19 & 65 & 56 & 61 \\
\hline Postoperative day 1 & 22 & 0.27 & 60 & 71 & 66 \\
\hline Postoperative day 7 & 20 & 0.58 & 69 & 87 & 78 \\
\hline Postoperative day 14 & 23 & 0.62 & 75 & 87 & 81 \\
\hline \multicolumn{6}{|l|}{ RIFLE } \\
\hline On admission & I category & 0.04 & 7 & 97 & 52 \\
\hline Postoperative day 1 & I category & 0.20 & 57 & 64 & 61 \\
\hline Postoperative day 7 & I category & 0.56 & 72 & 82 & 77 \\
\hline Postoperative day 14 & R category & 0.60 & 74 & 82 & 78 \\
\hline \multicolumn{6}{|l|}{ SOFA } \\
\hline On admission & 5 & 0.23 & 52 & 71 & 62 \\
\hline Postoperative day 1 & 11 & 0.41 & 77 & 64 & 71 \\
\hline Postoperative day 7 & 7 & 0.70 & 83 & 87 & 84 \\
\hline Postoperative day 14 & 7 & 0.69 & 85 & 84 & 84 \\
\hline \multicolumn{6}{|l|}{ Proteinuria plus SOFA } \\
\hline On admission & 5 & 0.26 & 57 & 70 & 63 \\
\hline Postoperative day 1 & 12 & 0.54 & 79 & 75 & 77 \\
\hline Postoperative day 7 & 8 & 0.72 & 85 & 85 & 85 \\
\hline Postoperative day 14 & 8 & 0.72 & 89 & 83 & 86 \\
\hline \multicolumn{6}{|c|}{ Proteinuria plus nonrenal SOFA } \\
\hline On admission & 5 & 0.26 & 54 & 76 & 63 \\
\hline Postoperative day 1 & 12 & 0.54 & 54 & 100 & 77 \\
\hline Postoperative day 7 & 8 & 0.68 & 86 & 82 & 84 \\
\hline Postoperative day 14 & 7 & 0.67 & 100 & 67 & 84 \\
\hline
\end{tabular}

Optimal cutoff points for predicting 3-month mortality were derived from receiver operator characteristic analysis. On admission (pre-transplant), post-transplant day 1, 7, and 14 , the Youden index and overall correctness for predicting 3-month mortality were higher for the proteinuria plus SOFA score than those for the proteinuria, Child-Pugh points, MELD score, RIFLE criteria, SOFA, and proteinuria plus nonrenal SOFA scores

Abbreviation: MELD model for end-stage liver disease, SOFA sequential organ failure assessment

power than SOFA alone (Table 6). Moreover, proteinuria plus SOFA also had the highest Youden index and the best overall correctness of prediction (Table 7).

AKI is a common complication in patients with decompensated liver disease, and its occurrence is correlated to poor prognosis [20]. Recently, several promising urinary protein biomarkers have been proved to be remarkably helpful for detecting AKI, such as Calprotectin, neutrophil gelatinase-associated lipocalin (NGAL), cystatin C, interleukin-18 (IL-18), kidney injury molecule-1 (KIM-1), and L-type or liver-type fatty acid-bind protein (LFABP) $[2,21-24]$. The presence of protein in urine might reflect structural or functional defects of the glomerular capillary barrier or the reabsorption receptors in the 

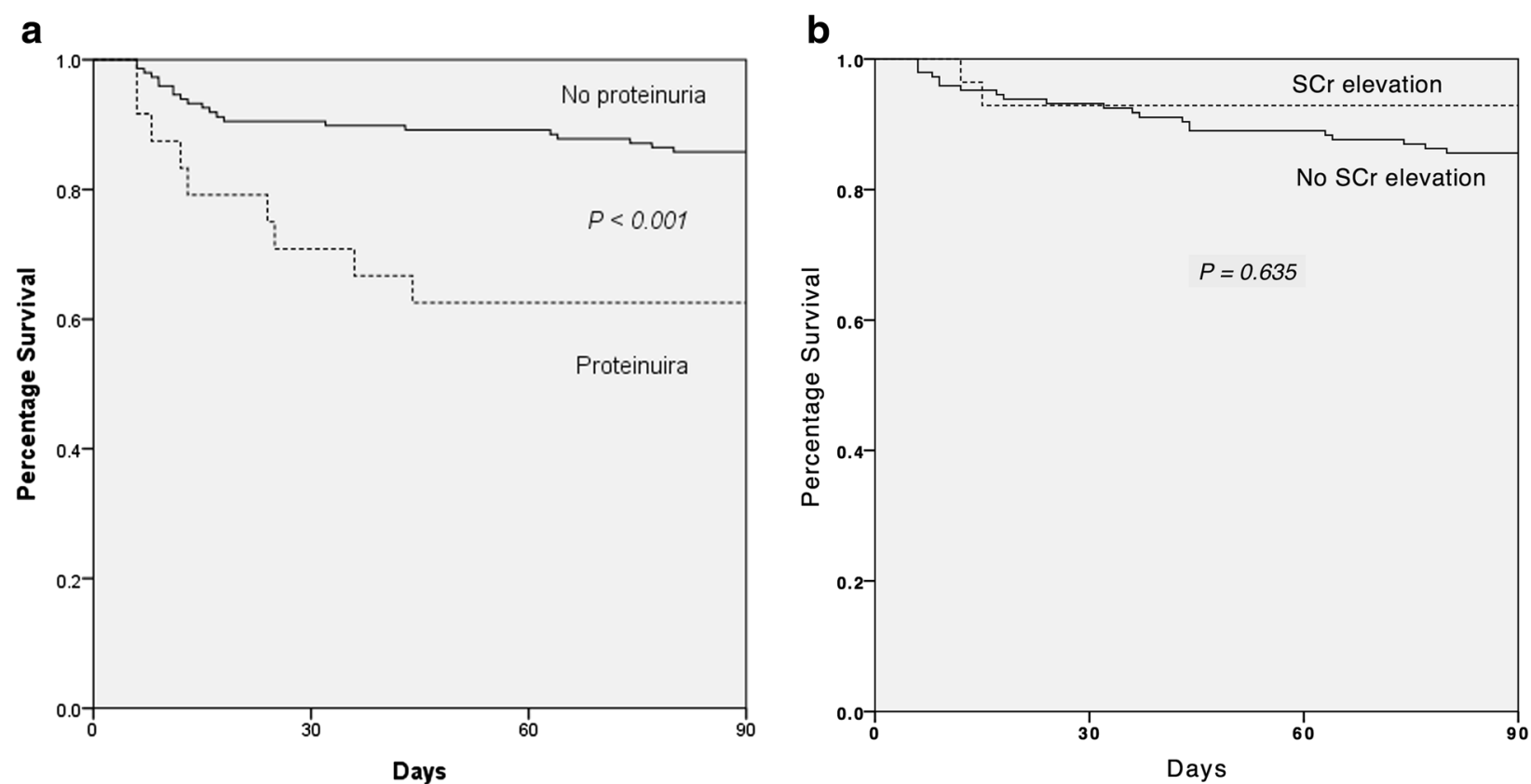

Fig. 1 Survival Functions Kaplan-Meier survival analysis in 323 patients according to the data measured before receiving liver transplantation. a Cumulative survival rates differed significantly for patients with proteinuria $(n=45)$ and those without proteinuria $(n=278)$ on the first day of ICU admission. b Cumulative survival rates did not differ significantly for patients with $\mathrm{SCr}$ level elevation (Increase in $\mathrm{SCr} \geq 1.5 \times$ baseline) $(n=28)$ and those without SCr elevation $(n=295)$ on the first day of ICU admission. ${ }^{*}$ Abbreviation: SCr, serum creatinine

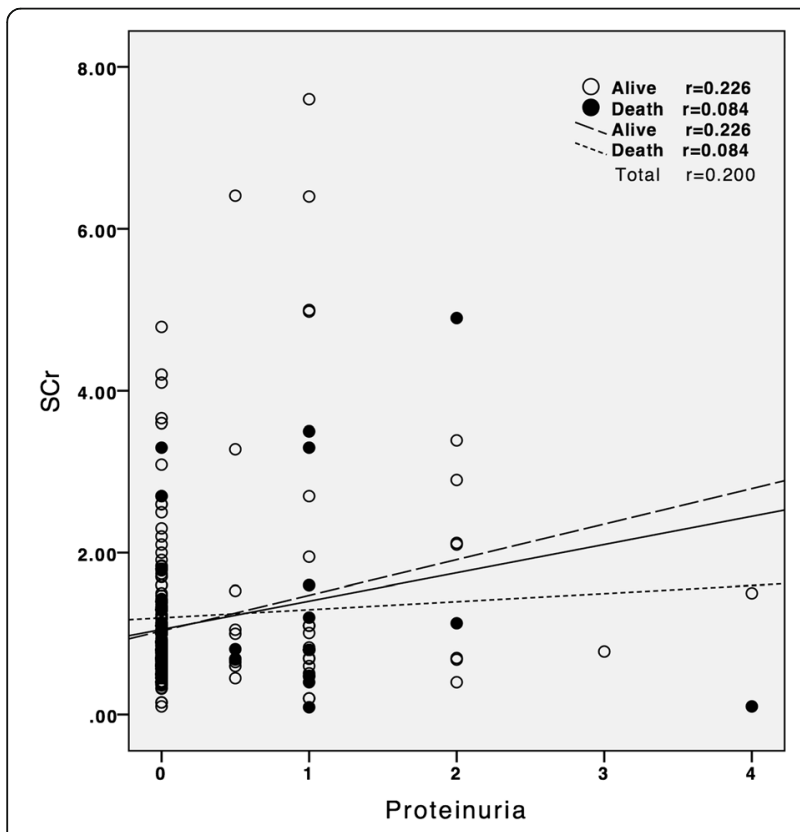

Fig. 2 Correlations of proteinuria and SCr measured on the first day of ICU admission for in-hospital mortality. The proteinuria is not correlated significantly $(p=0.143)$ with $\mathrm{SCr}$. *Abbreviation: $\mathrm{SCr}$, serum creatinine renal proximal tubules. Proteinuria is not only a sensitive indicator but also a risk factor for acute kidney injury [25]. Increasing evidence has indicated that proteinuria itself may activate intrarenal complement cascade, upregulate proximal tubular inflammatory and fibrogenic gene, trigger apoptotic response, and further lead to spreading of renal tubulointerstitial damage and adverse outcomes [26-31]. In the literature, urine albumin to creatinine ratio (UACR) is a precise method for measurement of albuminuria and identification of renal dysfunction [32, 33]. Nevertheless, previous study had documented that preoperative proteinuria could accurately predict the development of AKI in patients undergoing operation, irrespective of it is determined by UACR or urine dipstick analysis [34]. Previous reports also demonstrated that calculating urinary calprotectin/creatinine or NGAL/ creatinine ratio does not lead to higher prediction accuracy than using urinary calprotectin or NGAL alone [2, 24]. In this study, proteinuria was detected with the dipstick analysis, the advantages of this examination are inexpensive and easily performed and interpreted. Analyzed data showed presence of proteinuria on ICU admission (before transplantation) was correlated to increased risks of AKI, severe infection episodes, in-hospital mortality, and 90-day mortality (Fig. 1). The occurrence of AKI after transplantation, 

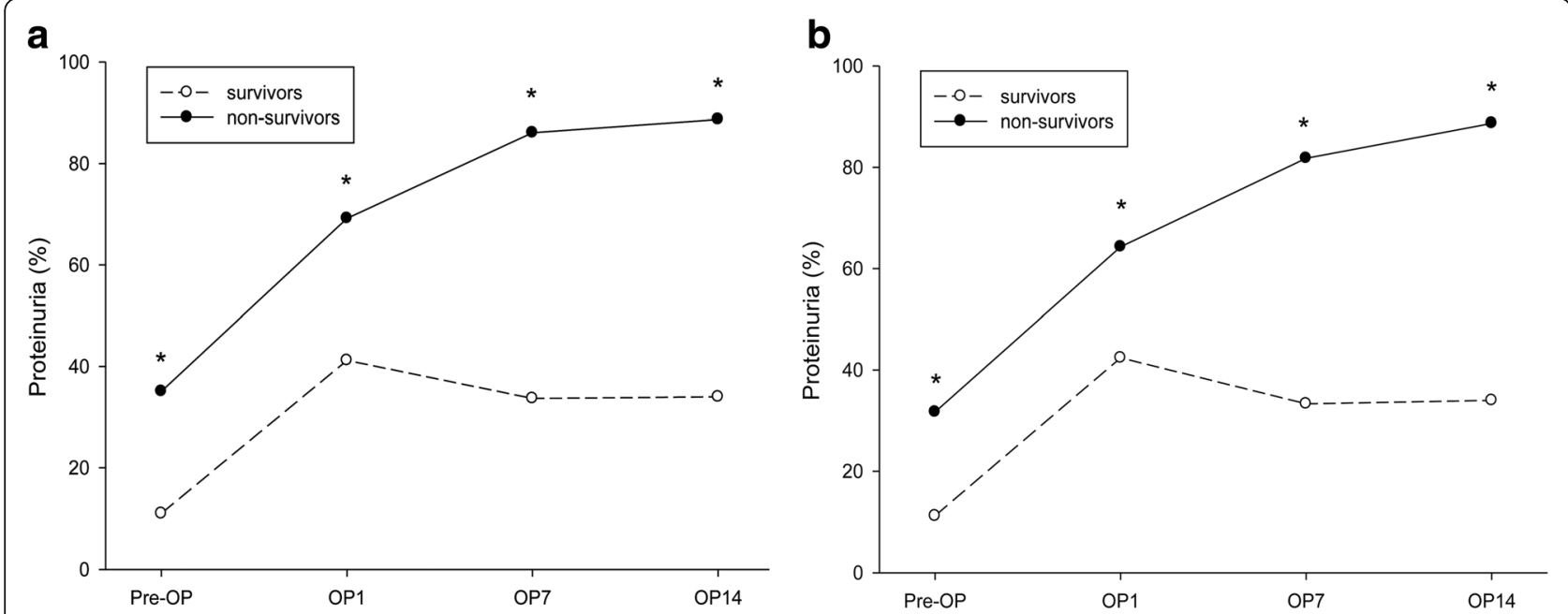

Fig. 3 The prevalence of proteinuria for the (a) in-hospital survival group (living, $n=281)$ and mortality group (dead, $n=42)$ and (b) 90-day survival group (living, $n=277$ ) and mortality group (dead, $n=46$ ) during the ICU admission day and postoperative days 1,7 , and 14. The prevalence of proteinuria significantly increased during this period among the in-hospital and 90-day mortality groups but not in the survivor groups $(* P<0.05)$

especially infection related AKI, was correlated to a markedly lower chance of survival (Table 3). Measurement of preoperative proteinuria might be useful to preemptively identify patients who have increased risk of AKI and severe infection episodes. Based on the observed results, it seems that avoiding nephrotoxic agents and choosing therapy carefully are crucial ways of renoprotection for patients present with proteinuria before transplant. More intensive postoperative care and infection prevention strategies, such as more aggressive prophylactic antimicrobial regimens and strict practice for infection prophylaxis, might also help to prevent further adverse outcomes for these patients. Further well-powered research is needed to study this issue.

Among patients undergoing liver transplant, development of renal dysfunction may be attributed to intraoperative caval cross-clamping with vascular outflow obstruction, reperfusion injury, significant infectious event, bleeding, perioperative hypotension with high vasopressor requirement, large-volume transfusions, exposure to nephrotoxic agents or use of calcineurin inhibitors [35-37]. As part of the acute phase response, general vascular permeability increases and the urinary excretion of protein and albumin occur within several hours after operation [38]. In our study, the decreaseddonor graft recipients who had prolonged cold ischemia time and the living-donor graft recipients who had longer warm ischemia time, were found to have significantly higher incidences of newly onset proteinuria on the first day after operation. These findings are consistent with previous studies [39-43]. Moreover, the prevalence of proteinuria decreased significantly within
7 days after operation in the in-hospital and 90-day survival groups but not in the mortality groups (Fig. 3). It implies that patients with poor physiologic adaptability are much more intolerable to the transient hemodynamic change of kidney. In addition, the persistence of proteinuria in serial assessment after liver transplantation might represent delayed recovery from the major operation and signify poor short-term prognosis.

Early detection of AKI after liver transplant could potentially retard the progression of renal dysfunction and prevent further adverse outcomes by prompt intervention [36, 44]. Many researches have shown that SOFA can completely present courses of major organ dysfunction and dynamic changes of illness severity after acute insults [45]. We have proposed that SOFA determined on postoperative day 7 has good predictive performance in short-term outcome of patients receiving liver transplant [8]. However, the renal parameter of the SOFA score, SCr concentration, does not seem to elevate until decline of renal function over than $50 \%$. This means that early detecting AKI by SOFA is hardly possible [36]. Our data revealed that absolute concentration and relative change of $\mathrm{SCr}$ levels are not significantly correlated to the presences of proteinuria and patient outcomes (Table 2, Figs. 1, 2), which highlights that $\mathrm{SCr}$ concentration is inaccurate for discovering kidney injury among patients with decompensated liver disease [46]. On the contrary, the occurrence of proteinuria might represent subtle renal function changes, and it also signifies generalized inflammatory environment and poor prognosis (correlation between proteinuria and CRP, before operation: $P<0.001$; postoperative 
day 1: $P=0.005)$. In this study, the combination of proteinuria improves the flaws of $\mathrm{SCr}$ and the prediction ability of SOFA during perioperative time, particularly on postoperative day 1 (an increase in the AUROC from 0.761 to 0.828 ) (Tables 6). These exciting findings seem to demonstrate that presence of proteinuria could provide outstanding early prognostic prediction for patients undergoing liver transplantation.

Despite the promising results obtained in this research, there are some possible limitation should be acknowledged. Firstly, this research was performed in just one tertiary medical center, so our results might not be exactly generalized to dissimilar patient population. Secondly, owing to the retrospective nature of our study, not every clinical factor was available. Thirdly, the patient population contained a high proportion of hepatitis B viral infection (34 \%) and may present as a special subgroup in the cirrhotic patients. Fourthly, predictive precision of logistic regression models is not perfect and flawless. Finally, the prognostic tools were applied on patients already admitted to the ICU, and were not used as a preadmission screening test, this might skew the analyses.

\section{Conclusions}

To conclude, this study showed that preoperative proteinuria is a significant risk factor of hospital mortality in patients undergoing liver transplantation. For patients exhibiting proteinuria before transplant, we suggest watch out for infection episodes, carefully choose therapy and do prompt intervention to prevent further adverse outcomes. Avoiding prolonged cold or warm ischemia time of transplantation could also reduce organ injury from reperfusion. The presence of proteinuria in serial assessment after liver transplantation has been proven to have early prognostic predictive effect and to assist the SOFA score with better discriminatory power in predicting short-term outcome. For these reasons, we recommend surveying the presence of proteinuria in preoperative and postoperative serial assessment. Proteinuria is supposed to be recognized as an important negative predictor for short-term survival of liver transplant patients.

\section{Abbreviations \\ AKl: Acute kidney injury; AUROC: Area under the receiver operating characteristic curve; CP points: Child-pugh points; ICU: Intensive care units; MELD: Model for end-stage liver disease; OP: Operation; RIFLE: The risk of renal failure, injury to the kidney, failure of kidney function, loss of kidney function, and end-stage renal failure; SCr: Serum creatinine; SOFA: Sequential organ failure assessment; TIPS: Transjugular intrahepatic portosystemic shunt; UACR: Urine albumin-creatinine ratio}

\section{Acknowledgements}

The authors thank the staff of the Core Lab and Liver Transplantation Team of the Chang Gung Memorial Hospital for technical assistance. We also express our sincere gratitude to all participants of the CGMH Critical Care Team.
Funding

None.

\section{Availability of data and materials}

The data that support the findings of this study are available from Liver Transplantation Center of the Chang Gung Memorial Hospital but restrictions apply to the availability of these data, which were used under license for the current study, and so are not publicly available. Data are however available from the authors upon reasonable request and with permission of Liver Transplantation Center of the Chang Gung Memorial Hospital.

\section{Authors' contributions}

YCC and HCP contributed to the conception and design of this research. HCP contributed to collection and interpretation of data. $\mathrm{HCP}, J P L$, and MJT contributed to manuscript drafting. WCL provided patient information and participated in the design and coordination. YJC, JPL, MJT, CCJ, MHT, PCF, CHC, MYC, YCT, CHC, CCH, JTF, and CWY contributed to offering intellectual content of the work and were involved in editing and revising the manuscript. All authors discussed and contributed to the final manuscript version.

\section{Competing interests}

The authors declare that they have no competing interests.

\section{Consent for publication}

Not applicable.

\section{Ethics approval and consent to participate}

This clinical investigation was approved by the Institutional Review Board of Chang Gung Memorial Hospital (approval No.101-2410B). The protocol of the study was designed in full compliance with Good Clinical Practice guidelines and the principles of the Declaration of Helsinki. In this study, no organs from executed persons were used. Because only preexisting data was examined, we did not obtrained written informed consent from each patient. Instead, patients were informed of their right for accepting or refusing enrollment by telephone interview. Before data analysis, the records of patients were anonymized and de-identified. The approaches are following ordinances concerning informed consent and enrollment expounded in guidelines.

\section{Author details}

${ }^{1}$ Department of Nephrology, Chang Gung Memorial Hospital, Keelung, Taiwan. ${ }^{2}$ Division of General Internal Medicine and Geriatrics Medicine, Chang Gung Memorial Hospital, Taipei, Taiwan. ${ }^{3}$ Chang Gung University College of Medicine, Taoyuan, Taiwan. ${ }^{4}$ Kidney Research Center, Department of Nephrology, Chang Gung Memorial Hospital, Taipei, Taiwan. ${ }^{5}$ Laboratory of Immunology, Department of General Surgery, Chang Gung Memorial Hospital, Taipei, Taiwan. ${ }^{6}$ Division of Gastroenterology, Chang Gung Memorial Hospital, Taipei, Taiwan.

Received: 16 December 2015 Accepted: 24 August 2016

Published online: 15 September 2016

\section{References}

1. Glassock RJ. Is the presence of microalbuminuria a relevant marker of kidney disease? Curr hypertens rep. 2010;12:364-8.

2. Heller F, Frischmann S, Grunbaum M, Zidek W, Westhoff TH. Urinary calprotectin and the distinction between prerenal and intrinsic acute kidney injury. Clin J Am Soc Nephrol. 2011;6:2347-55.

3. Huang T-M, Wu V-C, Young G-H, Lin Y-F, Shiao C-C, Wu P-C, et al. Preoperative proteinuria predicts adverse renal outcomes after coronary artery bypass grafting. J Am Soc Nephrol. 2011;22:156-63.

4. Lin LY, Jenq CC, Liu CS, Huang CS, Fan PC, Chang CH, et al. Proteinuria can predict short-term prognosis in critically ill cirrhotic patients. J Clin Gastroenterol. 2014;48:377-82.

5. Wehler M, Kokoska J, Reulbach U, Hahn EG, Strauss R. Short-term prognosis in critically ill patients with cirrhosis assessed by prognostic scoring systems. Hepatology. 2001;34:255-61.

6. Cholongitas E, Senzolo M, Patch D, Shaw S, Hui C, Burroughs AK. Review article: scoring systems for assessing prognosis in critically ill adult cirrhotics. Aliment Pharmacol Ther. 2006;24:453-64. 
7. Pan HC, Jeng CC, Tsai MH, Fan PC, Chang CH, Chang MY, et al. Scoring systems for 6-month mortality in critically ill cirrhotic patients: a prospective analysis of chronic liver failure - sequential organ failure assessment score (CLIF-SOFA). Aliment Pharmacol Ther. 2014;11:12953.

8. Wong CS, Lee WC, Jeng CC, Tian YC, Chang MY, Lin CY, et al. Scoring short-term mortality after liver transplantation. Liver transpl: official publication of the American Association for the Study of Liver Diseases and the International Liver Transplantation Society. 2010;16:138-46. doi:10.1002/lt.21969.

9. Fraley DS, Burr R, Bernardini J, Angus D, Kramer DJ, Johnson JP. Impact of acute renal failure on mortality in end-stage liver disease with or without transplantation. Kidney Int. 1998;54:518-24.

10. Barri YM, Sanchez EQ, Jennings LW, Melton LB, Hays S, Levy MF, et al. Acute kidney injury following liver transplantation: definition and outcome. Liver Transpl. 2009;15:475-83.

11. James MT, Hemmelgarn BR, Wiebe N, Pannu N, Manns BJ, Klarenbach SW, et al. Glomerular filtration rate, proteinuria, and the incidence and consequences of acute kidney injury: a cohort study. Lancet. 2010;376:2096-103.

12. Hsu RK, C-y H. Proteinuria and reduced glomerular filtration rate as risk factors for acute kidney injury. Curr Opin Nephrol Hypertens. 2011;20:211-7.

13. Levey A, Atkins R, Coresh J, Cohen E, Collins A, Eckardt K, et al. Chronic kidney disease as a global public health problem: approaches and initiatives-a position statement from Kidney Disease Improving Global Outcomes. Kidney Int. 2007;72:247-59.

14. Wiesner R, Edwards E, Freeman R, Harper A, Kim R, Kamath P, et al. Model for end-stage liver disease (MELD) and allocation of donor livers. Gastroenterology. 2003;124:91-6. doi:10.1053/gast.2003.50016.

15. Pan HC, Jenq CC, Lee WC, Tsai MH, Fan PC, Chang CH, et al. Scoring Systems for Predicting Mortality after Liver Transplantation. PLoS One. 2014;9:e107138.

16. Bellomo R, Ronco C, Kellum JA, Mehta RL, Palevsky P, Workgroup A. Acute renal failure - definition, outcome measures, animal models, fluid therapy and information technology needs: the Second International Consensus Conference of the Acute Dialysis Quality Initiative (ADQI) Group. Crit Care. 2004:8:R204-12. doi:10.1186/cc2872.

17. Youden W. Index for rating diagnostic tests. Cancer. 1950;3:32-5.

18. Akyildiz M, Karasu Z, Arikan C, Kilic M, Zeytunlu M, Gunsar F, et al. Impact of pretransplant MELD score on posttransplant outcome in living donor liver transplantation. Transplant Proc. 2004;36:1442-4. doi:10.1016/j.transproceed. 2004.05.004.

19. Leppke S, Leighton T, Zaun D, Chen SC, Skeans M, Israni AK, et al. Scientific Registry of Transplant Recipients: collecting, analyzing, and reporting data on transplantation in the United States. Transplant Rev (Orlando). 2013;27:50-6. doi:10.1016/j.trre.2013.01.002

20. Pan HC, Jenq CC, Tsai MH, Fan PC, Chang CH, Chang MY, et al. Risk models and scoring systems for predicting the prognosis in critically ill cirrhotic patients with acute kidney injury: a prospective validation study. PLoS One. 2012;7:7.

21. Coca SG, Yalavarthy R, Concato J, Parikh CR. Biomarkers for the diagnosis and risk stratification of acute kidney injury: a systematic review. Kidney Int. 2008:73:1008-16.

22. Chen YCFJ, Yang CW. Acute kidney injury biomarkers. Taiwan Crit Care Med 2009;10:121-8

23. Belcher JM, Sanyal AJ, Peixoto AJ, Perazella MA, Lim J, Thiessen-Philbrook $\mathrm{H}$, et al. Kidney biomarkers and differential diagnosis of patients with cirrhosis and acute kidney injury. Hepatology. 2014;60:622-32.

24. Chang $\mathrm{CH}$, Yang $\mathrm{CH}$, Yang $\mathrm{HY}$, Chen $\mathrm{TH}$, Lin $\mathrm{CY}$, Chang SW, et al. Urinary Biomarkers Improve the Diagnosis of Intrinsic Acute Kidney Injury in Coronary Care Units. Medicine. 2015;94:0000000000001703.

25. Abbate M, Zoja C, Remuzzi G. How does proteinuria cause progressive renal damage? J Am Soc Nephrol. 2006;17:2974-84.

26. Nangaku M, Pippin J, Couser WG. Complement membrane attack complex (C5b-9) mediates interstitial disease in experimental nephrotic syndrome. J Am Soc Nephrol. 1999;10:2323-31.

27. Morigi M, Macconi D, Zoja C, Donadelli R, Buelli S, Zanchi C, et al. Protein overload-induced NF-kB activation in proximal tubular cells requires $\mathrm{H}_{2} \mathrm{O} 2$ through a PKC-dependent pathway. J Am Soc Nephrol. 2002;13:1179-89.

28. Abbate M, Zoja C, Rottoli D, Corna D, Tomasoni S, Remuzzi G. Proximal tubular cells promote fibrogenesis by TGF-\&bgr; 1-mediated induction of peritubular myofibroblasts. Kidney Int. 2002;61:2066-77.

29. Nangaku M, Pippin J, Couser WG. C6 mediates chronic progression of tubulointerstitial damage in rats with remnant kidneys. J Am Soc Nephrol. 2002;13:928-36.
30. Tang S, Leung JC, Abe K, Chan KW, Chan LY, Chan TM, et al. Albumin stimulates interleukin-8 expression in proximal tubular epithelial cells in vitro and in vivo. J Clin Investig. 2003;111:515-27.

31. Erkan E, Garcia CD, Patterson LT, Mishra J, Mitsnefes MM, Kaskel FJ, et al. Induction of renal tubular cell apoptosis in focal segmental glomerulosclerosis: roles of proteinuria and Fas-dependent pathways. J Am Soc Nephrol. 2005;16:398-407.

32. Ciavarella A, Silletti A, Forlani G, Morotti L, Borgnino L, D'Apote M, et al. A screening test for microalbuminuria in type 1 (insulin-dependent) diabetes. Diabetes Res Clin Pract. 1989;7:307-12.

33. Eknoyan G, Levin NW. K/DOQI clinical practice guidelines for chronic kidney disease: evaluation, classification, and stratification. Am J Kidney Dis. 2002;39:S1-S266.

34. Coca SG, Jammalamadaka D, Sint K, Thiessen Philbrook H, Shlipak MG, Zappitelli $\mathrm{M}$, et al. Preoperative proteinuria predicts acute kidney injury in patients undergoing cardiac surgery. J Thorac Cardiovasc Surg. 2012;143:495-502.

35. Cabezuelo JB, Ramirez P, Rios A, Acosta F, Torres D, Sansano T, et al. Risk factors of acute renal failure after liver transplantation. Kidney Int. 2006;69:1073-80.

36. Wagener G, Minhaz M, Mattis FA, Kim M, Emond JC, Lee HT. Urinary neutrophil gelatinase-associated lipocalin as a marker of acute kidney injury after orthotopic liver transplantation. Nephrol Dial Transplant. 2011:26:1717-23.

37. Nastos C, Kalimeris K, Papoutsidakis N, Tasoulis M-K, Lykoudis PM, Theodoraki K, et al. Global Consequences of Liver Ischemia/Reperfusion Injury. Oxidative Med Cell Longev. 2014;2014:13. doi:10.1155/2014/906965.

38. Gosling P, Shearman CP, Gwynn BR, Simms MH, Bainbridge ET. Microproteinuria: Response to operation. Br Med J (Clin Res Ed). 1988;296:338

39. Paugam-Burtz C, Kavafyan J, Merckx P, Dahmani S, Sommacale D, Ramsay $M$, et al. Postreperfusion syndrome during liver transplantation for cirrhosis: outcome and predictors. Liver Transpl. 2009:15:522-9.

40. Lee HT, Park SW, Kim M, D'Agati VD. Acute kidney injury after hepatic ischemia and reperfusion injury in mice. Lab Invest. 2009:89:196-208.

41. Heidenhain C, Pratschke J, Puhl G, Neumann U, Pascher A, Veltzke-Schlieker W, et al. Incidence of and risk factors for ischemic-type biliary lesions following orthotopic liver transplantation. Transpl Int. 2010;23:14-22.

42. Wang B, Zhang Q, Zhu B, Cui Z, Zhou J. Protective effect of gadolinium chloride on early warm ischemia/reperfusion injury in rat bile duct during liver transplantation. PLoS One. 2013;8:14

43. Xia Y, Gong JP. Impact of recombinant globular adiponectin on early warm ischemia-reperfusion injury in rat bile duct after liver transplantation. Sci Rep. 2014:4:6426.

44. Yalavarthy $\mathrm{R}$, Edelstein $\mathrm{CL}$, Teitelbaum I. Acute renal failure and chronic kidney disease following liver transplantation. Hemodial Int. 2007;11:S7-S12

45. Goldhill DR, Sumner A. Outcome of intensive care patients in a group of British intensive care units. Crit Care Med. 1998;26:1337-45.

46. Pan HC, Chien YS, Jenq CC, Tsai MH, Fan PC, Chang CH, et al. Acute Kidney Injury Classification for Critically III Cirrhotic Patients: A Comparison of the KDIGO, AKIN, and RIFLE Classifications. Sci Rep. 2016;6:23022. doi:10.1038/ srep23022.

\section{Submit your next manuscript to BioMed Central and we will help you at every step:}

- We accept pre-submission inquiries

- Our selector tool helps you to find the most relevant journal

- We provide round the clock customer support

- Convenient online submission

- Thorough peer review

- Inclusion in PubMed and all major indexing services

- Maximum visibility for your research

Submit your manuscript at www.biomedcentral.com/submit 